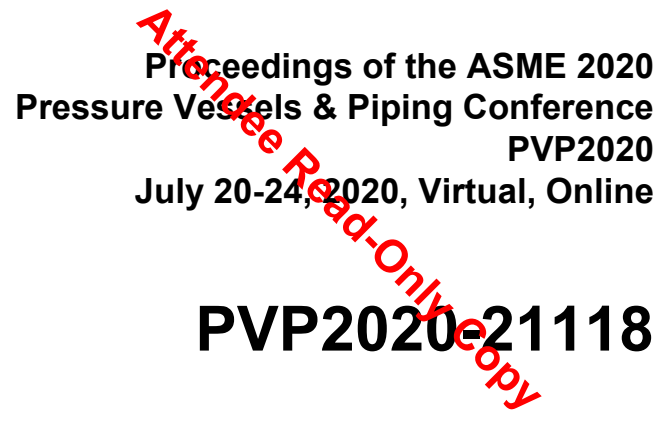

\title{
STRUCTURAL DYNAMIC MODIFICATION OF CYLINDRICAL SHELLS WITH VARIABLE THICKNESS
}

\author{
Farid Mahboubi Nasrekani ${ }^{1}$, Shymal Shivneel \\ Kumar, Sumesh Narayan. \\ Faculty of Science, Technology and Environment, \\ School of Engineering and Physics, The University of \\ the South Pacific (USP), Suva, Fiji.
}

\begin{abstract}
In this paper, the effects of some geometrical parameters on dynamic behavior of cylindrical shells with constant and variable thickness are studied. The equation of motion for the shell with constant thickness is extracted based on classical shell theory using Hamilton's principle. These equations which are a system of coupled partial differential equations are solved analytically and the natural frequency is determined for cylindrical shells with constant thickness. The natural frequency for cylindrical shells with variable thickness is determined using finite element method by employing ANSYS. The results are compared and the effect of different geometric parameters such as length, thickness, and radius on natural frequency is discussed. The specific ranges for geometric parameters have been determined in which there is no significant difference between shells with constant or variable thickness. Cylindrical shells with variable thickness have better stress and strain distribution and optimum weight, in compare with the shells with constant thickness and it is important to know in which ranges of dimensions and geometrical parameters, there are some significant differences between their mechanical properties such as natural frequency. The results are compared with some other references.

Keywords: Dynamic modification, Cylindrical shells, Variable thickness, Finite element method, Natural frequency.
\end{abstract}

\section{INTRODUCTION}

Shell structures have very extended applications in different industries, such as pressure vessels, aerospace, marine, liquid storage tanks, and etc. In particular, the optimization of shells is interesting with regards to conserving weight. Shells with variable thickness in comparison with shells with constant thickness have better characteristics in terms of stress and strain distribution, stability, and weight distribution. In previous years, numerous researches have been done, considering the buckling vibration of shells with variable thickness using KirchhoffLove's first approximation theory [1-5]. They investigated the effect of the variation of thickness on the critical parameters such as natural frequency and buckling load, using numerical methods such as Frobenius series method. Sofiyev and Erden [6] studied the stability of non-homogeneous cylindrical shells with variable thickness under a uniform external pressure which is a power function of time. The governing equations which were derived using Donnell stability equations, have been solved by employing the Galerkin's method. Aksogan and Sofiyev [7] investigated the dynamic buckling of an elastic cylindrical shell with varying thickness under a uniform external pressure. The governing equations have been extracted using Donnell stability equations and they have been solved by applying the Galerkin's method. Abbas et al. [8] presented a theoretical method to investigate the feasibility of using the transfer matrix method for open-variable thickness circular cylindrical shells under a hightemperature field. The governing equations were extracted in matrix form and they have been solved by employing fourthorder Runge-Kutta method. Grigorenko et al. [9] investigated the dynamic behavior of open cylindrical shells with variable thickness using the spline-collocation method and the method of discrete orthogonalization. Bahrami Ataabadi et al. [10] studied the vibrational behavior of orthotropic cylindrical shells with variable thickness. The governing equations have been determined using linear shell theory and the natural frequencies and mode shapes were obtained numerically. Fan et al. [11] presented an analytical method to investigate dynamic buckling load for a shell with variable thickness subjected to uniform external pressure based on the Donnell equations. Mahboubi Nasrekani and Eipakchi [12-15] investigated the effect of

${ }^{1}$ Contact author: farid.mn83@gmail.com,nasrekani_f@usp.ac.fj 
thickness variation and geometrical parameters on stability and buckling analysis of cylindrical shells with variable thickness.

In this study, the effects of geometrical parameters such as radius, thickness, and length, on dynamic behavior of cylindrical shells with constant and variable thickness are discussed. The equations of motion for the shell with constant thickness are extracted based on classical shell theory and using the Hamilton's principle. According to the classical shell theory, the radial displacements of each layer of shell are the same or it is independent to thickness. These equations which are a system of coupled partial differential equations are solved analytically and the natural frequencies are determined for cylindrical shells with constant thickness. The natural frequency for cylindrical shells with variable thickness is determined using finite element method by employing ANSYS. For the shells with constant and variable thickness, it is important to know in which ranges of dimensions, there are some significant differences between the mechanical properties such as natural frequency. Four different cases of thickness are considered in this paper. The results are compared with some other references.

\section{EQUATION OF MOTION}

The position of an element on the cross section of a shell in cylindrical coordinate system can be defined by three parameters $r, x$ and $\theta$ as FIGURE 1 . The origin of the coordinate system is on the mid-surface which is shown by dashed line. We have $r=R_{m}+z$; where $R_{m}$ is the mid-surface radius and $z$ is measured from the mid-surface of the shell. The cross-sectional areas of the shell for different cases are shown in FIGURE 1. For different cases, $R_{\text {Oout }}$ and $R_{\text {Oin }}$ are outer and inner radius at $x=0$ and $R_{\text {lout }}$ and $R_{\text {lin }}$ are outer and inner radius at $x=L$, respectively. $L$ is the length of shell in $x$ direction.
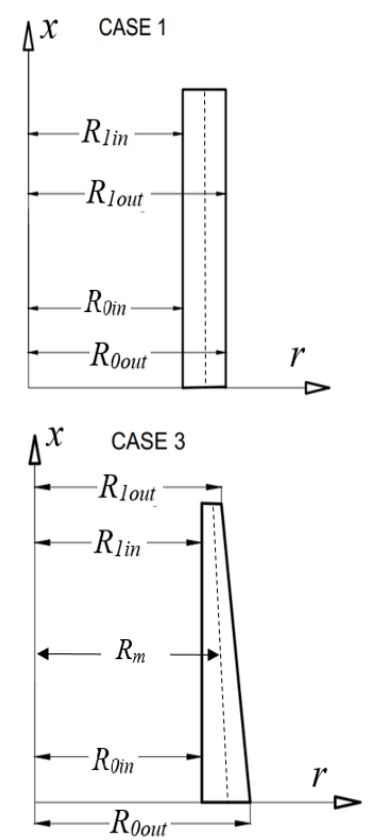
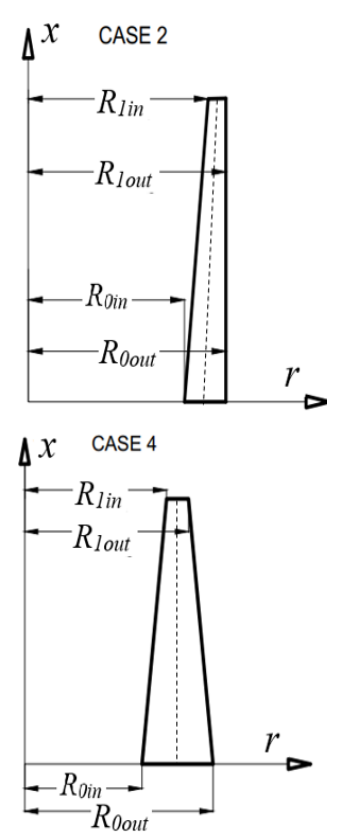

FIGURE 1: Cross-sectional area of different shells
According to the classical shell theory, the displacement filed for axisymmetric conditions is defined as following [16]:

$$
\begin{aligned}
& u(x, z, t)=u_{0}(x, t)-z \frac{\partial w_{0}(x, t)}{\partial x} \\
& w(x, z, t)=w_{0}(x, t) ; v=0
\end{aligned}
$$

where $u, v$, and $w$, are displacements in $x, \theta$, and $z$ directions respectively. $u_{0}$ and $w_{0}$ are the mid-surface displacements and they are unknown functions of $x$ and $t$. The nonzero strains components are [15]:

$$
\varepsilon_{x}=\frac{\partial u}{\partial x}=\frac{\partial u_{0}}{\partial x}-z \frac{\partial^{2} w_{0}}{\partial x^{2}} ; \varepsilon_{\theta}=\frac{w}{r}=\frac{w_{0}}{R_{m}+z}
$$

The stress-strain relations according to Hooke's law are [16]:

$$
\sigma_{x}=\frac{E}{1-v^{2}}\left(\varepsilon_{x}+v \varepsilon_{\theta}\right) ; \sigma_{\theta}=\frac{E}{1-v^{2}}\left(v \varepsilon_{x}+\varepsilon_{\theta}\right)
$$

$E$ and $v$ are the Young's modulus and Poisson's ratio, respectively. The Strain energy $U$, and kinetic energy $T$ are as the following:

$$
\begin{aligned}
& U=\frac{1}{2} \iiint\left(\sigma_{x} \varepsilon_{x}+\sigma_{\theta} \varepsilon_{\theta}\right) d V \\
& T=\frac{1}{2} \iiint \rho\left[\left(\frac{\partial u}{\partial t}\right)^{2}+\left(\frac{\partial w}{\partial t}\right)^{2}\right] d V \\
& d V=\left(R_{m}+z\right) \cdot d z \cdot d \theta \cdot d x \\
& -\frac{h}{2} \leq z \leq \frac{h}{2} ; 0 \leq \theta \leq 2 \pi ; 0 \leq x \leq L
\end{aligned}
$$

where $\rho$ is density. The stress resultants are obtained as:

$$
\begin{aligned}
& N_{x}=\int_{-h / 2}^{h / 2} \sigma_{x}\left(1+\frac{z}{R_{m}}\right) d z ; N_{\theta}=\int_{-h / 2}^{h / 2} \sigma_{\theta} d z \\
& M_{x}=\int_{-h / 2}^{h / 2} \sigma_{x}\left(1+\frac{z}{R_{m}}\right) z d z ; M_{\theta}=\int_{-h / 2}^{h / 2} \sigma_{\theta} z d z
\end{aligned}
$$

According to Hamilton's principle which states $\delta \int(T-U) d t=0$ and by substituting equations (4) and (5), the equations of motion as a function of stress resultants are extracted as following:

$$
\begin{aligned}
& \frac{\partial\left(R_{m} N_{x}\right)}{\partial x}-\rho R_{m} h \frac{\partial^{2} u_{0}}{\partial t^{2}}+\frac{\rho h^{3}}{12} \frac{\partial^{3} w_{0}}{\partial x \partial t^{2}}=0 \\
& \frac{\partial^{2}\left(R_{m} M_{x}\right)}{\partial x^{2}}-N_{\theta}-\frac{\rho}{12} \frac{\partial^{3}\left(h^{3} u_{0}\right)}{\partial x \partial t^{2}}-\rho R_{m} h \frac{\partial^{2} w_{0}}{\partial t^{2}} \\
& +\frac{\rho h^{3}}{12} \frac{\partial^{2}}{\partial x \partial t}\left(R_{m} \frac{\partial^{2}}{\partial x \partial t} w_{0}\right)=0
\end{aligned}
$$

By substituting equations (1) - (3) into equation (5), and by using these terms, the equation of motion are obtained as a function of displacement. These equations are not reported here. 


\section{ANALYTICAL PROCEDURE}

The response of equations of motion for shell with constant thickness is considered as $\left\{u_{0}, w_{0}\right\}=\{V(x)\}_{2 *_{1}} \exp (i \omega t)$, where $\omega$ is the axisymmetric natural frequency and by substituting into equation of motion it results in:

$$
\begin{aligned}
& {\left[B_{5}\right] \frac{d^{4} V}{d x^{4}}+\left[B_{4}\right] \frac{d^{3} V}{d x^{3}}+\left[B_{3}\right] \frac{d^{2} V}{d x^{2}}} \\
& +\left[B_{2}\right] \frac{d V}{d x}+\left[B_{1}\right]=\{0\}_{2 * 1} \\
& {\left[B_{1}\right]=\left[B_{6}\right]+i\left(\omega\left[B_{7}\right]-\omega^{3}\left[B_{9}\right]\right)} \\
& -\omega^{2}\left[B_{8}\right]+\left[B_{0}\right]
\end{aligned}
$$

Where $\left[B_{j}\right], j=0,1, \ldots, 8$ are the coefficient matrices. Equations (8), are a system of ordinary differential equations (ODE) and the response can be obtained as $\{V(x)\}=\{A\} \exp (\alpha x)$, which $\{A\}_{2 * 1}$ is the eigenvectors and $\alpha$ is the eigenvalues. By substituting $\{V(x)\}$ into equations (8), a system of algebraic equation as $[\mathrm{eq}]_{2 * 2}\{\mathrm{~A}\}_{2 * 1}=\{0\}$ will be obtained. For nonzero solution, the determinant of [eq] should be equated to zero which it results in a relation between $\alpha$ and $\omega$. It is an algebraic equation from order six with respect to $\alpha$. It has six roots or eigenvalues, and for each eigenvalue, there is an eigenvector $\{A\}$ which all of them are functions of $\omega$. So, the general solution of equations (8) is as the following:

$$
\{V\}=\sum_{j=1}^{6} C_{j}\{A\}_{j} e^{\alpha_{j} x}
$$

Where $C_{j}$, are constant coefficients and will be obtained from boundary conditions. By applying the boundary conditions at $\mathrm{x}=0$ and $\mathrm{L}$, six new algebraic equations are extracted as functions of $C_{j}, j=1 . .6$. For non-trivial response, we consider that, determinant of the coefficient matrix is equal to zero. It is a complicated algebraic equation and they are solved by employing the bisection method and the natural frequencies are determined.

\section{NUMERICAL METHOD}

To solve the equations of motion for the cylindrical shell with variable thickness, ANSYS FE software has been employed. To obtain the axisymmetric frequencies and mode shapes, PLANE82, in axisymmetric mode is used. This is an element with eight nodes and two translational degrees of freedom at each node. The cross section of the shell is modeled in ANSYS and the analysis is done in two dimension to determine the results [12].

FIGURE 2 shows mesh pattern for a specific model for case 2 with variable thickness and selected element. After modelling, creating the meshed structure, and applying the boundary conditions, the modal analysis for the shell has been performed to obtain the natural frequency and mode shapes.

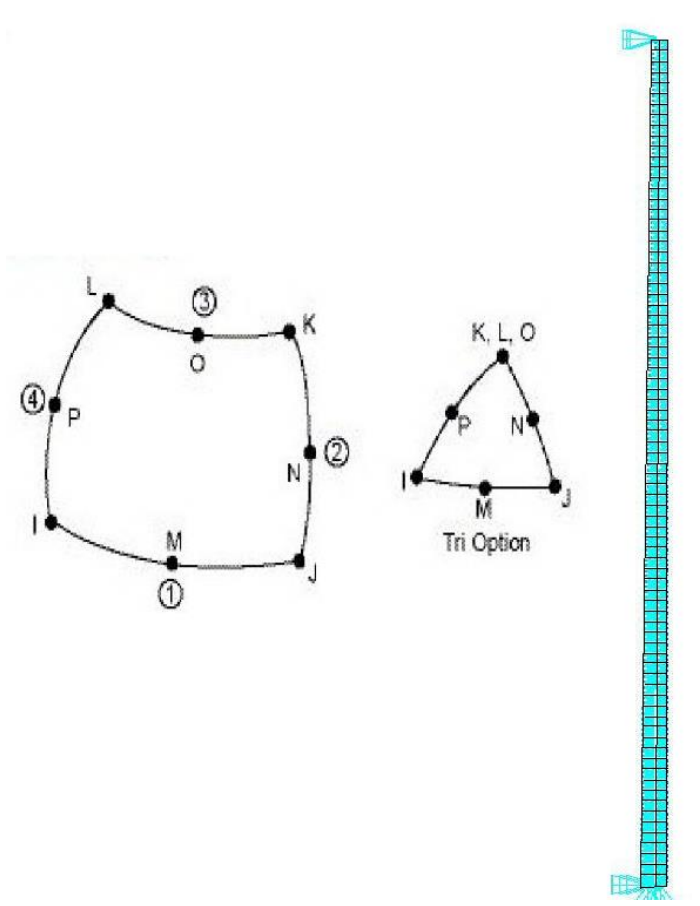

FIGURE 2: Mesh pattern and PLANE 82 element

\section{RESULTS AND DISCUSSION}

According to the presented method, a mathematical code has been prepared on Maple 15 mathematical environment to investigate the effects of different geometrical parameters on the natural frequencies. The boundary conditions are considered simply supported at both edges. In this section, the basic material properties of the shells are assumed as follows: $E=200 \mathrm{GPa}, \rho$ $=7800 \mathrm{~kg} / \mathrm{m}^{3}, v=0.3$.

FIGURE 3 shows the axisymmetric natural frequency for elastic cylindrical shells with constant thickness for various length to mid-radius ratio $\left(L / R_{m}\right)$, which is obtained from the presented analytical method, FE and presented method by Amabili [17]. Amabili obtained the equation of motion by employing the Donnell's theory. It is observed that, by increasing $L / R_{m}$, the natural frequency decreases.

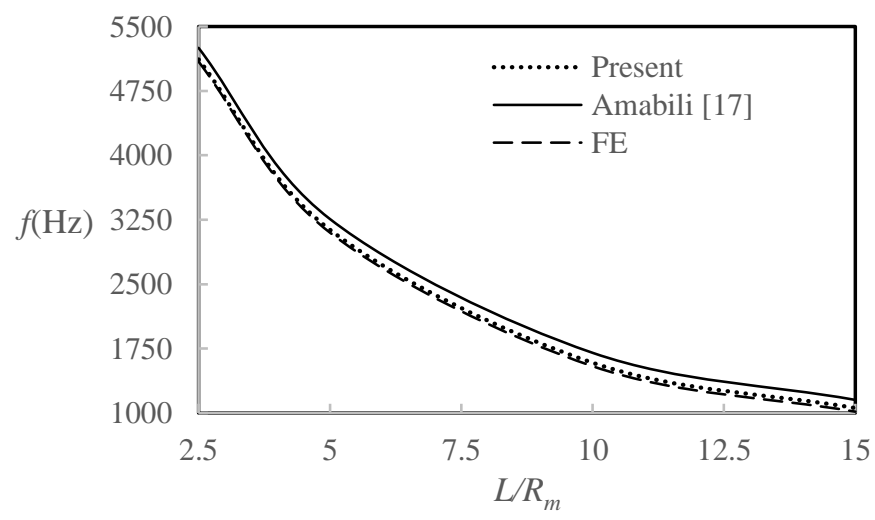

FIGURE 3: Comparison of the results of presented analytical, FE, and Amabili formula for different $L / R_{m}$ ratio $\left(R_{m} / h=16, R_{m}=0.16 \mathrm{~m}\right)$ 
As given in TABLE 1, the results for shells with constant thickness, are in a good agreement with the analytical results of Rao [18] based on the classical shell theory. It is seen that, by increasing the $L / R_{m}$ ratio, the natural frequency decreases.

TABLE 1: Comparison of natural frequency $(\mathrm{Hz})$ of shells with constant thickness for different $L / R_{m}$ ratio $\left(R_{m} / h=10, R_{m}=0.16 \mathrm{~m}\right)$.

\begin{tabular}{ccccc}
\hline \hline$L / R_{m}$ & 5 & 10 & 15 & 20 \\
\hline \hline Present & 3132.81 & 1584.03 & 1056.82 & 792.62 \\
\hline Rao [17] & 3132.95 & 1584.24 & 1056.95 & 792.74 \\
\hline
\end{tabular}

FIGURE 4 shows the difference between the first axisymmetric natural frequencies of different cases versus length to mid-radius ratio $\left(L / R_{m}\right)$. It is seen that, for $L / R_{m}>6$, there is no significant difference between the cases. In the other words, in terms of natural frequency, by increasing the length, there is no different between the different cases.

For instance, if we want to design an optimum pressure vessel of a certain capacity with minimum material and maximum natural frequency for presented data in TABLE 2, the correct $L / R_{m}$ ratio is determined 7.5 and according to FIGURE 4, shells with variable thickness will be the best option [19]. In this range of $L / R_{m}$ ratio, the natural frequency of shells with variable thickness is almost equal to the natural frequency of shells with constant thickness while due to the special geometrical form, shells with variable thickness have better weight, stress and strain distribution in comparison with shells with constant thickness. It should be mentioned that, the volume of different cases in FIGURE 4, is the same in each column.

TABLE 2: Design data.

\begin{tabular}{ccccc}
\hline \hline $\begin{array}{c}P \text { (Design } \\
\text { pressure, } \\
\text { psi) }\end{array}$ & $\begin{array}{c}\mathrm{C} \\
\text { (Corrosion } \\
\text { allowance, } \\
\text { in) }\end{array}$ & $\begin{array}{c}\text { S (Stress } \\
\text { value of } \\
\text { material, } \\
\text { psi) }\end{array}$ & $\begin{array}{c}\text { E (Joint } \\
\text { efficiency) }\end{array}$ & $\begin{array}{c}\mathrm{V} \\
\text { (Vessel } \\
\text { volume, } \\
\text { cu.ft) }\end{array}$ \\
\hline 100 & 0.0625 & 16000 & 0.80 & 1000 \\
\hline
\end{tabular}

FIGURE 5 shows the effect of mid-radius to thickness ratio on the first axisymmetric natural frequency of different cases. As a result, for $R_{m} / h>24$, the difference between the natural frequencies of different cases is negligible. Moreover, by increasing both $R_{m} / h$ and $L / R_{m}$ ratios, the natural frequency decreases. In these figures, the $R_{m} / h$ and $L / R_{m}$ ratios are calculated at $x=L / 2$ and all the cases have the same volume. Since, for the shells with large size of length and radius, their natural frequencies for different cases are in the same range and the shells with variable thickness have better characteristics in terms of stress and strain distribution, and stability, it can be concluded that, the shells with variable thickness have better applications in these ranges of dimensions.

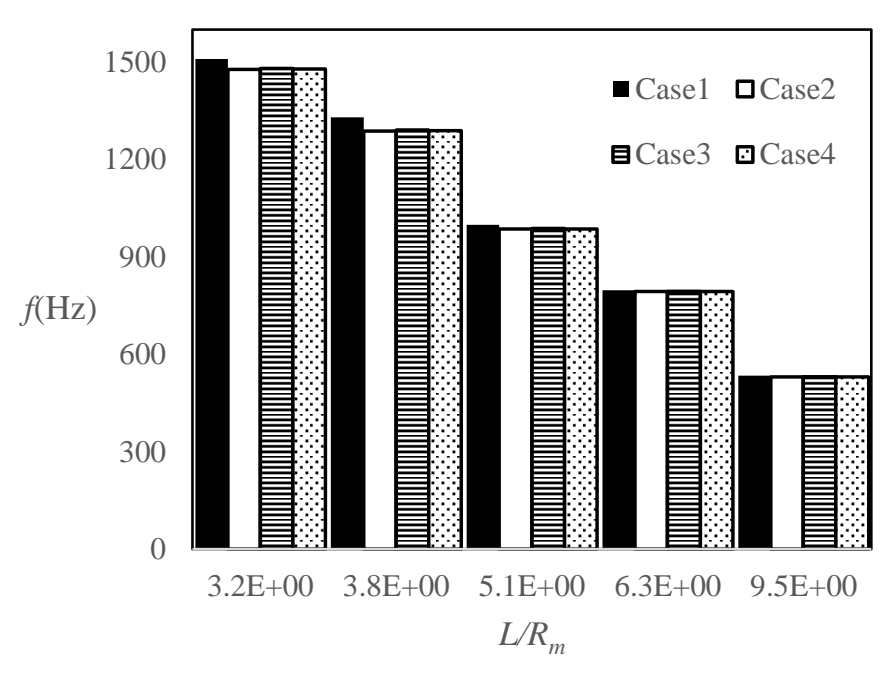

FIGURE 4: Effect of length to mid-radius ratio on differences between the first axisymmetric natural frequency for different cases ( $h$ $($ at $x=L / 2)=0.015 \mathrm{~m}$ )

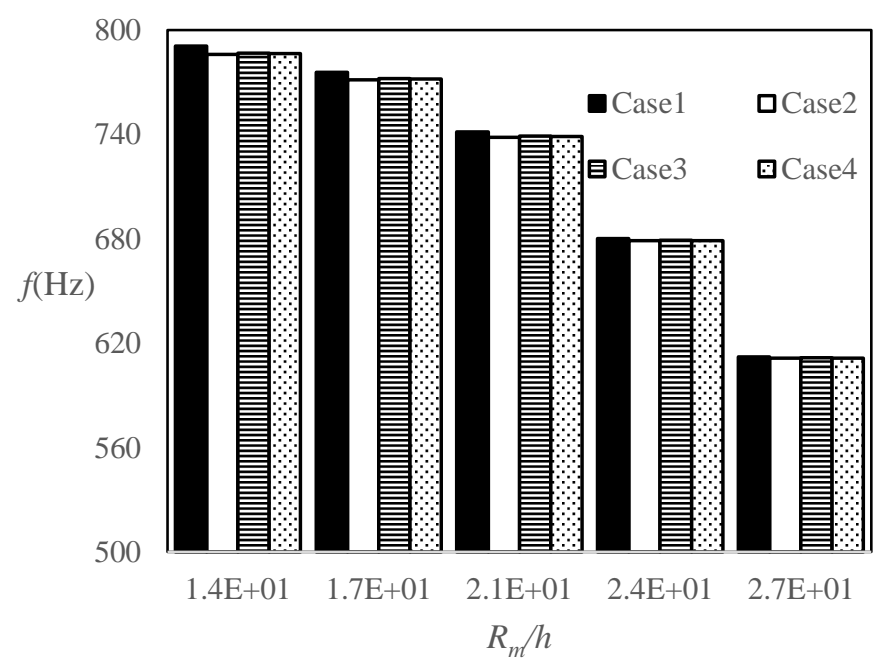

FIGURE 5: Effect of mid-radius to thickness ratio on differences between the first axisymmetric natural frequency for different cases ( $L$ $=1 \mathrm{~m})$

For the cases 2 and 3 in FIGURE 1, we define the gradient of thickness as following:

$$
\alpha=\left|\frac{R_{1 \text { in }}-R_{0 \text { in }}}{L}\right|_{\text {case } 2} \text { or }\left|\frac{R_{\text {lout }}-R_{0 \text { out }}}{L}\right|_{\text {case } 3}
$$

FIGURE 6, shows the effect of the parameter $\alpha$ on natural frequency. It is seen, by increasing the gradient of thickness, the natural frequency decreases significantly. In this figure, all of the shells have the same volume. 


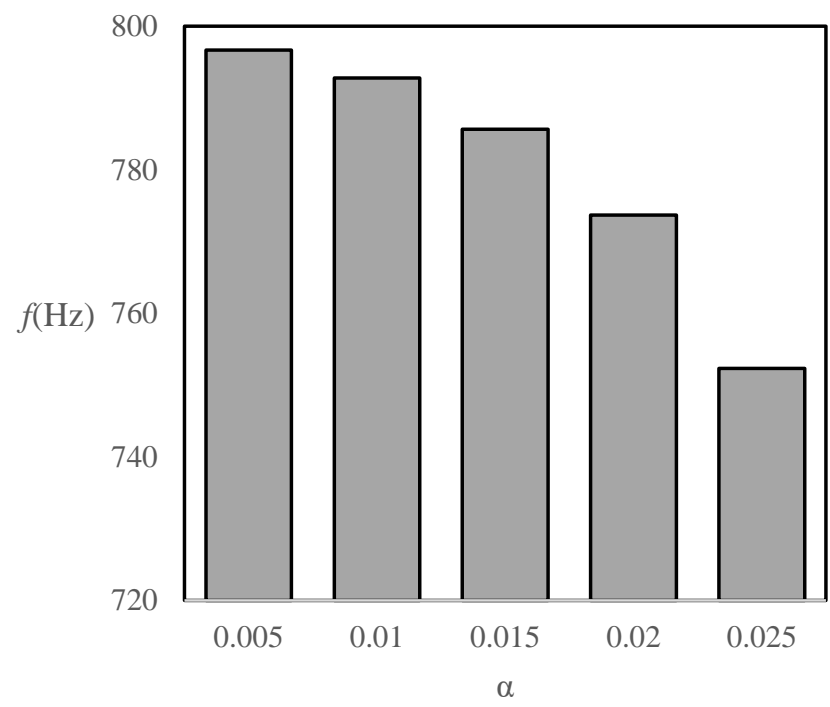

FIGURE 6: Effect of the parameter $\alpha$ on the first axisymmetric natural frequency $\left(L=1 \mathrm{~m}, R_{m}(\right.$ at $x=L / 2)=0.1575 \mathrm{~m}, h($ at $\left.x=L / 2)=0.015 \mathrm{~m}\right)$

\section{CONCLUSION}

In order to study the differences between dynamic behavior of four different cases of cylindrical shells with constant and variable thickness, an investigation is conducted.

- $\quad$ By increasing $L / R_{m}$ ratio, the natural frequency for all cases decreases and for $L / R_{m}>6$ there is no significant difference between different cases in terms of natural frequency.

- $\quad$ By increasing $R_{m} / h$ ratio, the natural frequency for all cases decreases and for $R_{m} / h>24$ there is no significant difference between different cases in terms of natural frequency.

- $\quad$ For the ranges $L / R_{m}>6$ and $R_{m} / h>24$, the shells with variable thickness are preferable to the shells with constant thickness. Because they have better weight, stress and strain distribution.

- For cases 2 and 3, by defining the gradient of thickness parameter, it has been concluded that by increasing this parameter, the natural frequency decreases.

In this paper the results for the shells with finite length is presented which these results can be extended for shells with infinite length or pipes. In addition, these results can be useful for pressure vessels as an application. The extracted results determine some ranges of dimensions which in them, there are some significant differences between the natural frequencies. As a designer, it is important to know, which type of shell for which range of dimension is more suitable. Designers can use extracted results in addition to the data reported from pressure vessel handbooks. For example when $R_{m} / h$ ratio is more than 24 , it is better to use shells with variable thickness. Moreover, it should be mentioned that the presented results are for the studied range of material properties.

\section{REFERENCES}

[1] Tonin, R. F. and Bies, D. A., 1979, "Free vibration of circular cylinders of variable thickness", Journal of Sound and Vibration, Vol. 62, pp165-180.

[2] Irie, T., Yamada, G., and Kaneko Y., 1981, "Free vibration of a conical shell with variable thickness", Journal of Sound and Vibration, Vol. 82, pp83-94.

[3] Suzuki, K., Konno, M., Kosawada, T., and Tkahashi, S., 1982, "Axi-symmetric vibrations of a vessel with variable thickness", Bulletin of the Japan Society of Mechanical Engineering, Vol. 25, No. 208, pp1591-1600.

[4] Tkahashi, S., Suzuki, K., and Kosawada, T., 1985, "Vibrations of conical shells with varying thickness", Bulletin of the Japan Society of Mechanical Engineering, Vol. 28, pp117123.

[5] Koiter, W. T., Elishakoff, I., Li, Y. W., and Starness, J. H., 1994, "Buckling of an axially compressed cylindrical shell of variable thickness", International Journal of Solids and Structures, Vol. 31, pp797-805.

[6] Sofiyev, A. H., Erden, H., 2002, "The stability of nonhomogeneous elastic cylindrical thin shells with variable thickness under a dynamic external pressure", Turkish Journal of Engineering and Environmental Sciences, Vol. 26, No. 2, pp155165.

[7] Aksogan, O., Sofiyev, A. H., 2002, "Dynamic buckling of a cylindrical shell with variable thickness subject to a timedependent external pressure varying as a power function of time", Journal of Sound and Vibration, Vol. 254, No. 4, pp693702.

[8] Abbas, L. K., Lei, M., Rui, X., 2010, "Natural vibrations of open-variable thickness circular cylindrical shells in high temperature field", Journal of Aerospace engineering, Vol. 23, No. 3.

[9] Grigorenko, A. Y., Efimova, T. L., Sokolova, L. V., 2012, "On the investigation of free vibrations of nonthin cylindrical shells of variable thickness by the spline-collocation method", Journal of Mathematical Sciences, Vol. 181, No. 4, pp506-519.

[10] Bahrami Ataabadi, P., Khedmati, M. R., Bahrami Ataabadi, M., 2014, "Free vibration analysis of orthotropic thin cylindrical shells with variable thickness by using spline functions", Latin American Journal of Solids and Structures, Vol. 11, pp2099-2121.

[11] Fan, H. G., Chen, Z. P., Feng, W. Z., Zhou, F., and Cao, G. W., 2015, "Dynamic buckling of cylindrical shells with arbitrary axisymmetric thickness variation under time dependent external pressure", International Journal of Structural Stability and Dynamics, Vol. 15, No. 3, 1450053.

[12] Mahboubi Nasrekani, F. Eipakchi, H. R., 2015, "Nonlinear analysis of cylindrical shells with varying thickness and moderately large deformation under nonuniform compressive pressure using the first-order shear deformation theory", Journal of Engineering Mechanics (ASCE), Vol. 141, No. 5. 
[13] Mahboubi Nasrekani, F. Eipakchi, H. R., 2017, "An analytical procedure for buckling load determination of cylindrical shells with variable thickness using first order shear deformation theory", AUT Journal of Mechanical Engineering, Vol. 1, No. 2, pp211-218.

[14] Mahboubi Nasrekani, F. Eipakchi, H. R., 2019, "Analytical solution for buckling analysis of cylinders with varying thickness subjected to combined axial and radial loads", International Journal of Pressure Vessels and Piping, Vol. 172, pp220-226.

[15] Mahboubi Nasrekani, F. Eipakchi, H. R., 2019, "Axisymmetric buckling of cylindrical shells with non-uniform thickness and initial imperfection", International Journal of Steel Structures, Vol. 19, No. 2, pp443-445.

[16] Sadd, M. H., 2009, "Elastic theory, application, and numeric". Elsivier Inc, UK.

[17] Amabili, M., 2008, "Nonlinear vibration and stability of shells and plates". Cambridge University Press, New York.

[18] Rao, s. s., 2007, "Vibration of continuous systems". Wiley, Hoboken.

[19] Megyesy, E. F., 2008, "Pressure vessel handbook". PV Publishing, INC., Oklahoma. 